\title{
Melatonin Attenuates White Matter Injury via Reducing Oligodendrocyte Apoptosis After Subarachnoid Hemorrhage in Mice
}

\author{
Dongdong LIU1,2,", Yushu DONG ${ }^{1, *}$, Gen $\mathrm{LI}^{1,2}$, Zheng ZOU11, Guangzhi HAO ${ }^{1}$, Hua FENG ${ }^{3}$, Pengyu PAN ${ }^{1}$, \\ Guobiao LIANG $^{1}$ \\ ${ }^{1}$ General Hospital of Northern Theater Command (General Hospital of Shenyang Military Command), Department of Neurosurgery, \\ Shenyang, China \\ ${ }^{2}$ Dalian Medical University, Dalian, Liaoning, China \\ ${ }^{3}$ Third Military Medical University (Army Medical University), Southwest Hospital, Department of Neurosurgery, Chongqing, China \\ *Both authors contribute equally to this article.
}

Corresponding author: Pengyu PAN, Guobiao LIANG panpengyu09@sina.com, liangguobiao6708@163.com

\section{ABSTRACT}

AIM: To determine whether melatonin (MLT) mitigates white matter (WM) injury by attenuating NOD-like receptor family pyrin domain-containing 3 (NLRP3)-associated oligodendrocyte apoptosis after subarachnoid hemorrhage (SAH).

MATERIAL and METHODS: SAH model C57BL/6J mice were created using an endovascular perforation technique. The mice were injected intraperitoneally with MLT at doses of $50 \mathrm{mg} / \mathrm{kg}, 150 \mathrm{mg} / \mathrm{kg}$ and $300 \mathrm{mg} / \mathrm{kg}$. The animals were evaluated for neurological outcomes according to neurological score, brain water content, myelin degradation, amyloid precursor protein (APP) accumulation, apoptosis, and levels of NLRP3, caspase-1, interleukin-1 $\beta, \mathrm{Bcl}-2$, and Bcl-2-interacting mediator of cell death (Bim) expression after $\mathrm{SAH}$.

RESULTS: MLT at a dose of $50 \mathrm{mg} / \mathrm{kg}$ improved neurological score, alleviated brain edema, and reduced WM injury. In addition, loss of myelin basic protein (MBP) and accumulation of APP, and expression in the ipsilateral/left hemisphere were found after SAH, and were reversed by MLT treatment. NLRP3 signal activation was found in microglia and apoptosis in oligodendrocytes were observed after SAH. MLT reduced oligodendrocyte apoptosis by regulating Bim and Bcl-2.

CONCLUSION: Results of this study indicated that MLT exerts a WM-protective effect in SAH pathophysiology, possibly by attenuating apoptosis in oligodendrocytes.

KEYWORDS: Subarachnoid hemorrhage, White matter, Oligodendrocyte, Melatonin, Apoptosis, Mice

ABBREVIATIONS: ANOVA: Analysis of variance, APP: Amyloid precursor protein, BBB: Blood-brain barrier, Bcl-2: B cell leukemia/ lymphoma 2, Bim: Bcl-2-interacting mediator of cell death, EBI: Early brain injury, IL-1ß: Interleukin-1 $\beta$, MBP: Myelin basic protein, MLT: Melatonin, PBS: Phosphate-buffered saline, SAH: Subarachnoid hemorrhage, WM: White matter.

Dongdong LIU (1) : 0000-0002-4365-2178

Yushu DONG (10: :0000-0002-9287-5183

Gen LI (1) : 0000-0001-8005-6079
Zheng ZOU (1):0000-0002-8437-5584

Guangzhi HAO (1): 0000-0001-5236-9350

Hua FENG : 0000-0003-4489-9217
Pengyu PAN (D) : 0000-0001-8694-5483

Guobiao LIANG (D) : 0000-0001-6332-190X 


\section{INTRODUCTION}

$\bigcirc$ ubarachnoid hemorrhage (SAH) is commonly encountered in the clinic. It is usually caused by rupture of an aneurysm and is associated with high morbidity and mortality (20). A recent theory posited that early brain injury $(\mathrm{EBI})$ is the most promising target for treatment of SAH. EBI includes neuronal death, surrounding apoptosis, microcirculation dysfunction, disruption of the blood brain barrier (BBB), brain edema, and other pathophysiological mechanisms in the early stages of SAH (23). Approximately $50 \%$ of tissue in the human brain is white matter (WM), which contains neuronal axons and oligodendrocytes. However, acute WM injury has not been extensively studied in experimental models of SAH. Several clinical and experimental studies have indicated that WM may be involved in neurological outcomes after SAH $(7,11,16,18)$. Therefore, to alleviate WM injury after SAH and improve neurological deficits, it is critical to develop novel therapies.

Previous investigations have indicated that inflammation and apoptosis appear early and play a critical role in neurological deficits after SAH (2). NOD-like receptor family pyrin domain-containing 3 (NLRP3) has been considered to be a key factor in both bacterial and aseptic inflammation during pathophysiological processes, and involves activated downstream caspase-1/II-1 $\beta$ (25). Melatonin (MLT) has recently been found to protect and prevent neurological deterioration by reducing inflammation in preclinical experiments. Because MLT is amphipathic and easily passes through the BBB, we deliberated whether MLT could affect WM injury and attenuate EBI. The present study aimed to investigate the role of MLT in neurological impairment and pathophysiological changes in WM using an experimental mouse model of SAH. We hypothesized that MLT attenuates WM injury by preventing NLRP3-associated microglia activation and oligodendrocyte apoptosis. Our study aimed to produce robust and reliable data to support this hypothesis.

\section{MATERIAL and METHODS}

\section{Mice}

Animal operations were approved by the Ethic Committee of General Hospital of Northern Theater Command (General Hospital of Shenyang Military Command), and the procedures complied with the ARRIVE guidelines and the National Institutes of Health Guide for the Care and Use of Laboratory Animals (Decision date: Nov/1st/2017; Number: 2017024). In this study, 108 male C57BL/6J mice, weighing $22 \mathrm{~g}$ to 30 $\mathrm{g}$ (purchased from the Department of Experimental Animal of General Hospital of Shenyang Military Command), were randomly divided into 6 groups as follows: sham $(n=24) ; \mathrm{SAH}$ $(n=12), S A H+$ vehicle $(n=24) ; S A H+M L T 50 \mathrm{mg} / \mathrm{kg}(\mathrm{n}=12) ;$ $\mathrm{SAH}+\mathrm{MLT} 150 \mathrm{mg} / \mathrm{kg}(\mathrm{n}=24) ;$ and SAH + MLT 300/kg $(\mathrm{n}=12)$.

\section{SAH Model}

Murine SAH model were created using an endovascular perforation technique, similar to a method previously described (30). Briefly, mice were anesthetized by intraperitoneal (i.p.) injection of sodium pentobarbital $(40 \mathrm{mg} / \mathrm{kg}$ ), followed by exposure of the bifurcation of the common carotid artery using blunt dissection. The external carotid artery (ECA) was sheared distally to leave a stump, if possible. A 5-0 sharpened monofilament nylon suture was inserted from the stump of the ECA directly to the internal carotid artery (ICA) and pierced the intracranial bifurcation of the ICA terminal segment. During the surgical procedure, an electric blanket was placed under the animals maintain rectal temperature at $37^{\circ} \mathrm{C}$. Mice in the sham group underwent all of the same procedures but without perforation.

The extent of hemorrhage in the subarachnoid space was evaluated according to a previously described grading system (24). Cistern on the ventral surface of the brain was partitioned into 6 parts, each of which was scored from 0-3 for the level of blood clot. The total score was the sum of scores for the 6 areas (0-18 points). Exclusions were made when the total score was $<8$ without remarkable neurological impairment.

\section{Assessment of Neurological Deficit}

Neurological scores were obtained using a protocol incorporating the modified Garcia score and beam balance score at 24 hours $(h)(n=6)$ and $72 h(n=6)$ after SAH. These two scales have been previously described and validated (21). Two blinded observers evaluated mean neurological scores.

\section{Drug}

MLT (Sigma-Aldrich, Shanghai, China) $50 \mathrm{mg} / \mathrm{kg}, 150 \mathrm{mg} / \mathrm{kg}$, $300 \mathrm{mg} / \mathrm{kg}$, and vehicle (1\% alcohol) were intraperitoneally injected $15 \mathrm{~min}$ after induction of $\mathrm{SAH}$. Mice in the sham group were not injected.

\section{Brain Water Content}

Mice were euthanized $24 \mathrm{~h}$ and $72 \mathrm{~h}$ after $\mathrm{SAH}$, and brain specimens $(n=6)$ were carefully dissected away from the skull and quickly cut into bilateral hemispheres, cerebellum, and brain stem. The four separate parts were weighed on a small sheet of aluminum foil to measure wet weight (4). The four parts were dried at $57^{\circ} \mathrm{C}$ for $48 \mathrm{~h}$ to measure dry weight. Brain water content was calculated using the following equation:

Water content $=\frac{\text { wet weight-dry weight }}{\text { wet weight }} \times 100 \%$.

\section{Terminal Deoxynucleotidyl Transferase dUTP Nick End-labeling Staining}

The terminal deoxynucleotidyl transferase dUTP nick end labeling (TUNEL) assay was performed as previously described (3). Brain sections $(n=6)$ were stained using a commercially available kit according to manufacturers' recommendations (Roche, Mannheim, Germany). Briefly, slides were immersed in proteinase- $\mathrm{K}$ in Tris- $\mathrm{HCl}(10 \mathrm{mM})$ buffer at $37{ }^{\circ} \mathrm{C}$ for $15 \mathrm{~min}$, then rinsed with phosphate-buffered saline (PBS) and incubated in $0.3 \%$ hydrogen peroxide. The slides were then immersed in sodium citrate $(0.1 \%)$ and Triton X-100 $(0.1 \%)$ solution for 3 minutes. After 3 washes with PBS, the sections were incubated with the TUNEL reaction mixture, containing TdT and Cy3-dUTP, for $60 \mathrm{~min}$ at $37^{\circ} \mathrm{C}$. 


\section{Immunohistochemistry}

Fixed, frozen brain sections were subjected to immunofluorescence staining $(n=6)$ as previously described using the following primary antibodies: anti-MBP (1:500 dilution; Santa Cruz Biotechnology, TX, USA), anti-Iba-1, anti-amyloid precursor protein (APP), anti-interleukin (IL)-1 $\beta$, anti-CNPase, and anti-NeuN (1:500 dilution; Abcam, Cambridge, United Kingdom) (9). The slides were then examined using a fluorescence microscope (Zeiss, Oberkochen, Germany). Myelin basic protein (MBP) and APP per field were counted using ImageJ software. Brain sections were stained with Luxol fast blue (LFB) for myelin imaging $(n=1)$ in accordance with a previously described method (8).

\section{Western Blot}

The protocol used for Western blotting was similar to that described in the authors' previous work (32). Briefly, the corpus callosum and the internal capsule in the left hemisphere $(n=6)$ were isolated macroscopically. Proteins were detected using the following primary antibodies: anti-caspase-1, anti-NLRP3, anti-IL-1 $\beta$ (1:1000, Cell Signaling Technology, Boston, MA, USA), anti-Bcl-2, anti-Bcl-2-interacting mediator of cell death (Bim) (1:500; Abcam, Cambridge, United Kingdom). Anti- $\beta$ actin (1:1000; Santa Cruz Biotechnology, Santa Cruz, CA, USA) was used as an internal control.

\section{Statistical Analysis}

Data are expressed as mean \pm standard error of the mean (SEM). Comparison among the groups was performed using one-way analysis of variance (ANOVA) and the Tukey post hoc multiple comparisons test. All statistical evaluations were performed using GraphPad Prism version 6 (GraphPad Inc, San Diego, CA, USA); differences with $p<0.05$ were considered to be statistically significant.

\section{Ethics Approval and Consent to Participate}

All experimental procedures were approved by the Ethics Committee of General Hospital of Northern Theater Command (General Hospital of Shenyang Military Command) and performed in accordance with the guidelines in the National Institutes of Health Guide for the Care and Use of Laboratory Animals, and adhered to the ARRIVE guidelines (Animal Research: Reporting in Vivo Experiments, https://www.nc3rs. org.uk/arrive-guidelines).

\section{RESULTS}

\section{MLT Improved Neurological Function After SAH}

No mice in the sham group died; however, 1 in the SAH group, 3 in the SAH + vehicle group, and 2 in the SAH + MLT group (1 in the SAH+MLT $50 \mathrm{mg} / \mathrm{kg}$ group, 3 in the SAH + MLT $150 \mathrm{mg} /$ $\mathrm{kg}$ group, and 1 in the SAH + MLT $300 \mathrm{mg} / \mathrm{kg}$ group) died within $24 \mathrm{~h}$ after SAH on account of mass clot in the subarachnoid space. There were no significant differences in grading scores among the groups at $24 \mathrm{~h}$ and $72 \mathrm{~h}$ (Figure 1A, E).

To investigate whether MLT attenuated neurological deficits, mice in SAH and SAH + vehicle groups were evaluated using the modified Garcia and beam balance tests. At $24 \mathrm{~h}$ and 72 h after SAH, modified Garcia scores were clearly lower in the $\mathrm{SAH}$ and $\mathrm{SAH}+$ vehicle groups compared with mice in the sham group (Figure 1C, G). Neurological deficits were attenuated by MLT treatment at doses of $50 \mathrm{mg} / \mathrm{kg}, 150 \mathrm{mg} /$ $\mathrm{kg}$, and $300 \mathrm{mg} / \mathrm{kg}$ (Figure 1C, G). Furthermore, the beam balance test also indicated significant neurological impairment in the SAH and SAH + vehicle groups, which was alleviated by MLT treatment (Figure 1B, F).

\section{MLT Alleviated Brain Edema and WM Injury}

Brain edema can independently indicate poor neurological outcome after SAH. To investigate whether brain edema developed after SAH, the protective effect of MLT on WM in the MLT- and vehicle-treated groups were compared. Data revealed higher brain water content in the vehicle group than in the sham group in both hemispheres (Figure 1D, H). In addition, treatment with MLT $50 \mathrm{mg} / \mathrm{kg}, 150 \mathrm{mg} / \mathrm{kg}$, and $300 \mathrm{mg} / \mathrm{kg}$ effectively attenuated brain edema (Figure 1D, H). There was no significant difference among the $50 \mathrm{mg} / \mathrm{kg}, 150$ $\mathrm{mg} / \mathrm{kg}$ and $300 \mathrm{mg} / \mathrm{kg}$ doses. As such, a dose of $50 \mathrm{mg} / \mathrm{kg}$ was chosen for subsequent follow-up experiments.

Loss of myelin was found in the corpus callosum and internal capsule of the ipsilateral/left hemisphere in the SAH and SAH + vehicle groups at $24 \mathrm{~h}$, and was attenuated by MLT treatment at a dose of $50 \mathrm{mg} / \mathrm{kg}$ (Figure 2A). WM structures contain axons and myelin sheath. Axonal accumulation of APP is a classic marker of axonal injury indicative of cytoskeletal damage (14, 18). Demyelination can be reflected by a downtrend of MBP, a primary component of the myelin sheath (19). Mice in the $\mathrm{SAH}+$ vehicle group exhibited apparent decreases in MBP and accumulation of APP in the left hemisphere $24 \mathrm{~h}$ after SAH. MLT treatment $(50 \mathrm{mg} / \mathrm{kg})$, resulted in loss of MBP and APP accumulation were attenuated compared with the vehicle group (Figure 2C-F). Moreover, mice in the vehicle group exhibited more TUNEL-positive (apoptotic) cells compared with sham-treated mice. In contrast, MLT treatment $(50 \mathrm{mg} /$ $\mathrm{kg}$ ) eliminated apoptotic cells in WM areas (Figure 2G, H).

\section{MLT Attenuated NLRP3-Induced Apoptosis of Oligodendrocytes}

Apoptosis of neurons and oligodendrocytes induced by NLRP3 activation plays a vital role in the pathophysiology of SAH (10). Previously, the authors reported that MLT could suppress NLRP3 activation, which induces subsequent caspase-1 and IL-1 $\beta$ maturation (5). Under pathological conditions in the central nervous system, microglia act as a main source of NLRP3 production. Microglia accumulation in the WM and increasing numbers of apoptotic oligodendrocytes were observed after SAH (Figure 3A-D). After MLT delivery, microglia accumulation and oligodendrocyte apoptosis were attenuated. Moreover, protein levels of NLRP3, caspase-1, and IL-1 $\beta$ were elevated at $24 \mathrm{~h}$ post-SAH (Figure $4 \mathrm{~A}-\mathrm{D}$ ) and MLT treatment reversed these alterations. Furthermore, Western blot also confirmed elevation of $\mathrm{Bcl}-2$ and decreases in Bim (Figure 4E-G). These effects were reversed by treatment with MLT (50 mg/kg). 
(A)

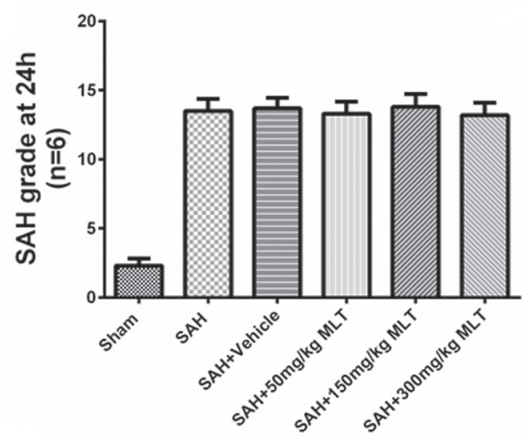

(C)

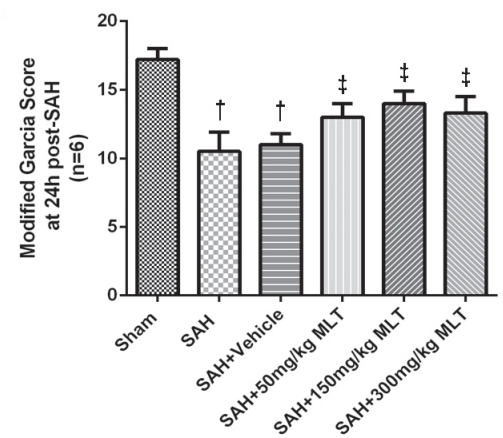

(E)

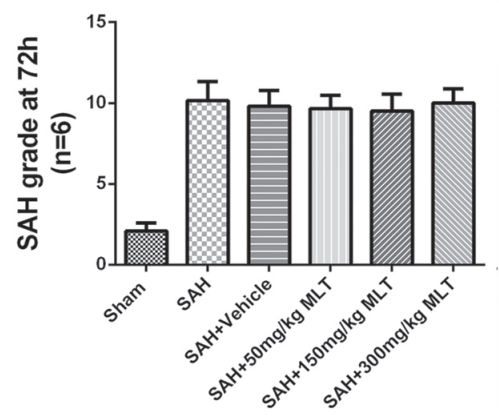

G

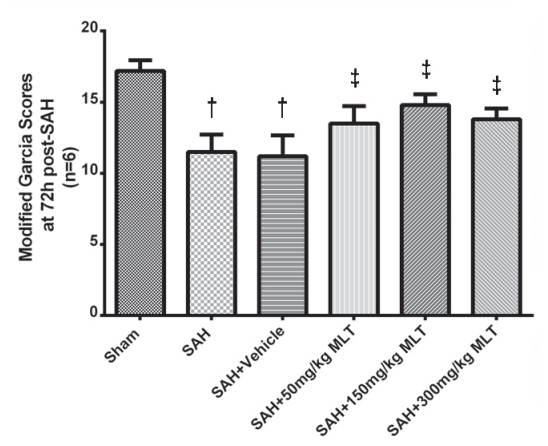

B
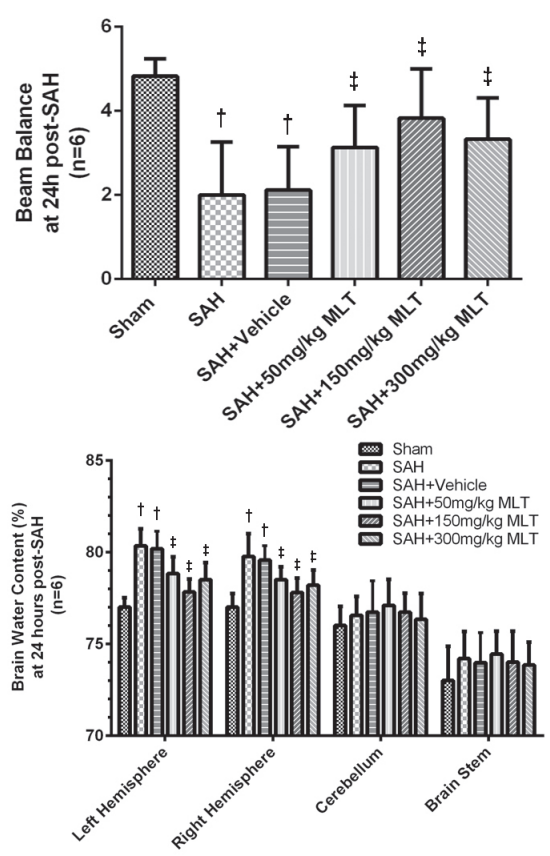

F

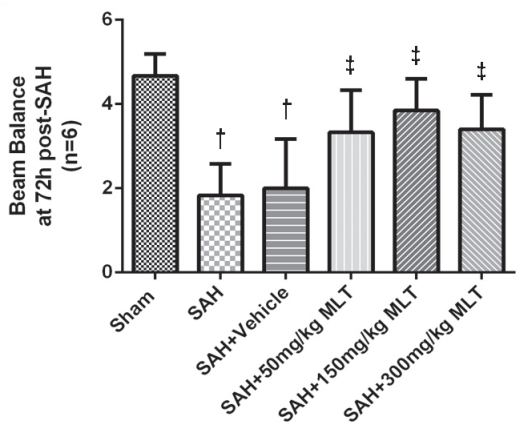

(H)

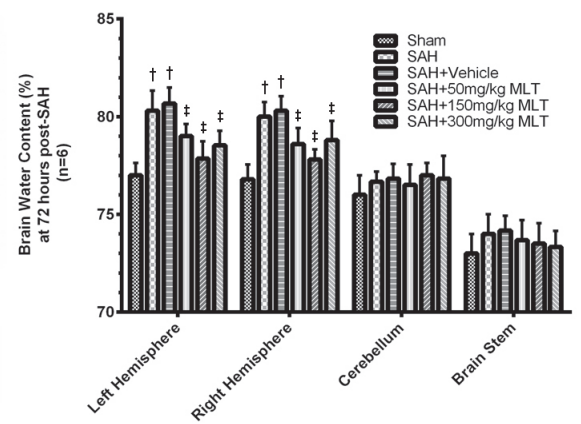

Figure 1: Neurological function assessment and brain edema by melatonin (MLT) treatment after subarachnoid hemorrhage (SAH). A) SAH grading scores revealed no significant differences among vehicle, MLT $50 \mathrm{mg} / \mathrm{kg}$, MLT $150 \mathrm{mg} / \mathrm{kg}$ and MLT $300 \mathrm{mg} / \mathrm{kg} 24 \mathrm{hours}$ (h) after SAH. B, C) Neurological tests showed significantly lower score in SAH and SAH + vehicle group compared with sham group. MLT $50 \mathrm{mg} / \mathrm{kg}, 150 \mathrm{mg} / \mathrm{kg}$, and $300 \mathrm{mg} / \mathrm{kg}$ treatment could increase the behavior text score significantly. Modified Garcia scores and Beam balance text revealed no different among $50 \mathrm{mg} / \mathrm{kg}, 150 \mathrm{mg} / \mathrm{kg}$, and $300 \mathrm{mg} / \mathrm{kg}$. D) Brain edema was observed at $24 \mathrm{~h}$ after SAH. In contrast to SAH and SAH + vehicle groups, MLT $50 \mathrm{mg} / \mathrm{kg}, 150 \mathrm{mg} / \mathrm{kg}, 300 \mathrm{mg} / \mathrm{kg}$ administration reduced brain water content efficiently. E) SAH grading scores demonstrated no significant differences among vehicle and MLT (50 mg/kg, $150 \mathrm{mg} / \mathrm{kg}$, and $300 \mathrm{mg} /$ kg) $72 \mathrm{~h}$ after SAH. F, G) At $72 \mathrm{~h}$ behavior test results were profoundly lower in the SAH and SAH + vehicle groups after SAH induction and reversed by treatment with MLT at doses of $50 \mathrm{mg} / \mathrm{kg}, 150 \mathrm{mg} / \mathrm{kg}$, and $300 \mathrm{mg} / \mathrm{kg}$. H) At $72 \mathrm{~h}$, brain edema was detected after SAH, but decreased by MLT treatment at doses of $50 \mathrm{mg} / \mathrm{kg}, 150 \mathrm{mg} / \mathrm{kg}$, and $300 \mathrm{mg} / \mathrm{kg}$ compared with the SAH and SAH + vehicle groups. 'Statistically significant difference (i.e., $\mathrm{p}<0.05)$ compared with the sham group; ‘Statistically significant difference (i.e., $p<0.05)$ compared with sham group, $\mathrm{SAH}+$ vehicle $(\mathrm{p}<0.05)$. 


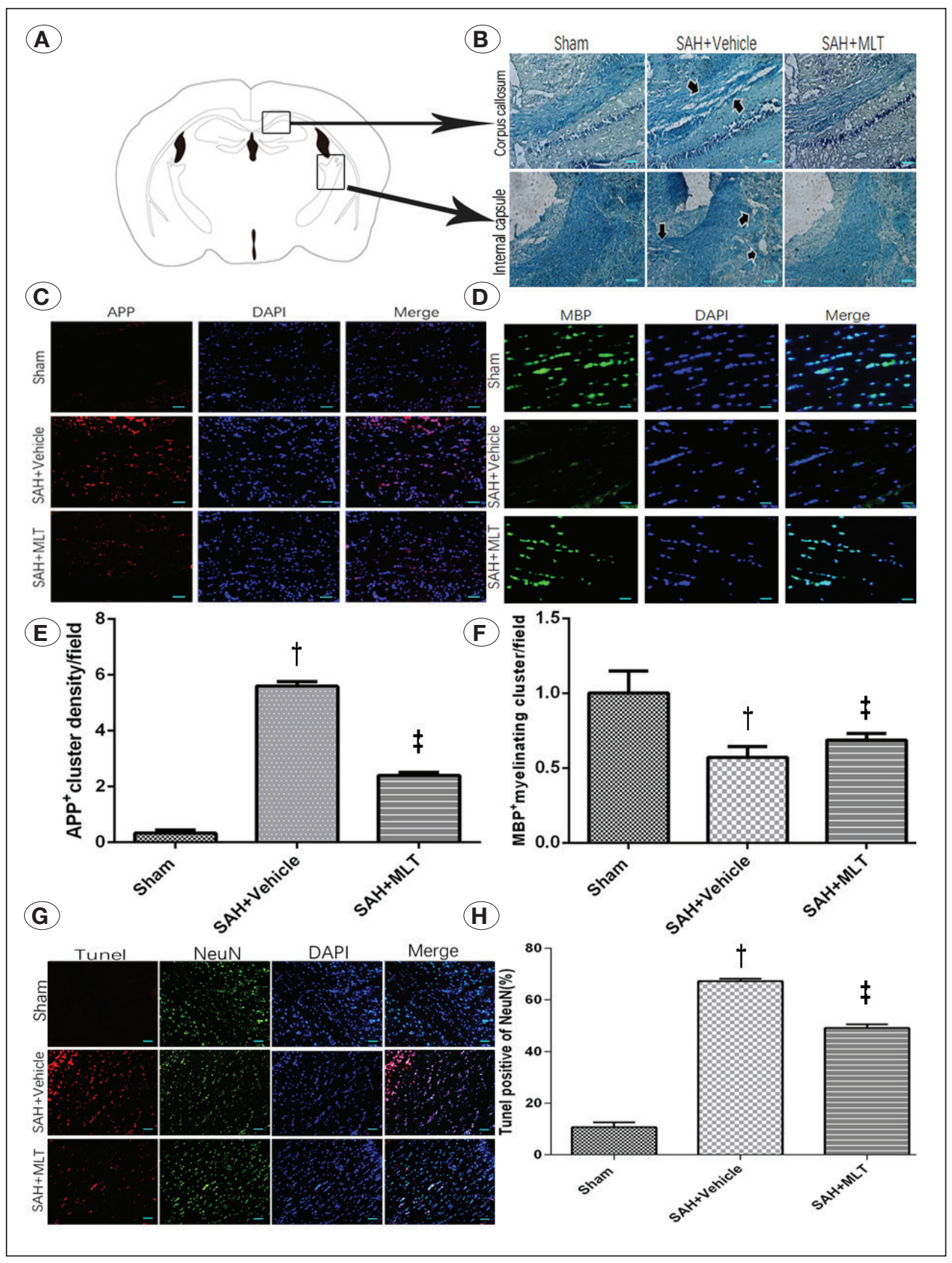

Figure 2: White matter (WM) injury and neuronal apoptosis after subarachnoid hemorrhage (SAH). A) Sketch map of brain coronary section and areas stained by Luxol fast blue (LFB).

B) LFB staining demonstrating marked disturbances in the WM of the left hemisphere 24 hours (h) after SAH in vehicle-treated SAH mice. After MLT injection (50 $\mathrm{mg} / \mathrm{kg})$, the disturbance was apparently attenuated in the ipsilateral hemisphere. Scale bar $=20 \mu \mathrm{m}$.

C-F) Immunofluorescence revealing loss of myelin basic protein (MBP) and accumulation of amyloid precursor protein (APP) $24 \mathrm{~h}$ after SAH in left WM area, which was reversed by MLT treatment. C, Scale bar $=20 \mu \mathrm{m}$; $\mathrm{D}$, Scale bar $=10 \mu \mathrm{m}$. G-H) Terminal deoxynucleotidyl transferase dUTP nick end labeling (TUNEL) staining of neurons increasing by $\mathrm{SAH}$ induction and reduced by MLT treatment $24 \mathrm{~h}$ after SAH. G, Scale bar $=20 \mu \mathrm{m}$. †Statistically significant difference (i.e., $\mathrm{p}<0.05$ ) compared with sham group;

₹Statistically significant difference (i.e., $\mathrm{p}<0.05$ ) compared with sham group; $\mathrm{SAH}+$ vehicle $\mathrm{p}<0.05$.

\section{DISCUSSION}

Results of the present study demonstrated improved neurological scores after treatment with MLT compared with vehicle in an experimental mouse model of SAH. We further demonstrated that MLT reduced brain edema and provided protection of the WM against acute injury in EBI. Additionally, these phenomena were associated with NLRP3-induced WM injury and neuronal apoptosis. As we demonstrated, MBP was downregulated and APP accumulated after SAH but were reversed by treatment with $\mathrm{MLT}(50 \mathrm{mg} / \mathrm{kg})$. These findings suggest that MLT demonstrates promising potential in treating $\mathrm{EBI}$ after SAH.
Various pathophysiological changes, such as the mass effect of clot, increasing intracranial pressure, decompensation of cerebral autoregulation, spreading depolarization, BBB breakdown, release of toxic blood components, secondary inflammation, and cell death occur immediately after hemorrhage (13). These injuries contribute to poor neurological outcome(s); however, few studies have focused on WM injury after SAH (22). The effect of WM fiber injury on neurological deficits, consciousness, and memory after SAH may be overlooked due to the paucity of WM in the brains of rodents, which are the most common experimental animals (17). Results of a study using a mouse model of SAH suggested that the WM is particularly 


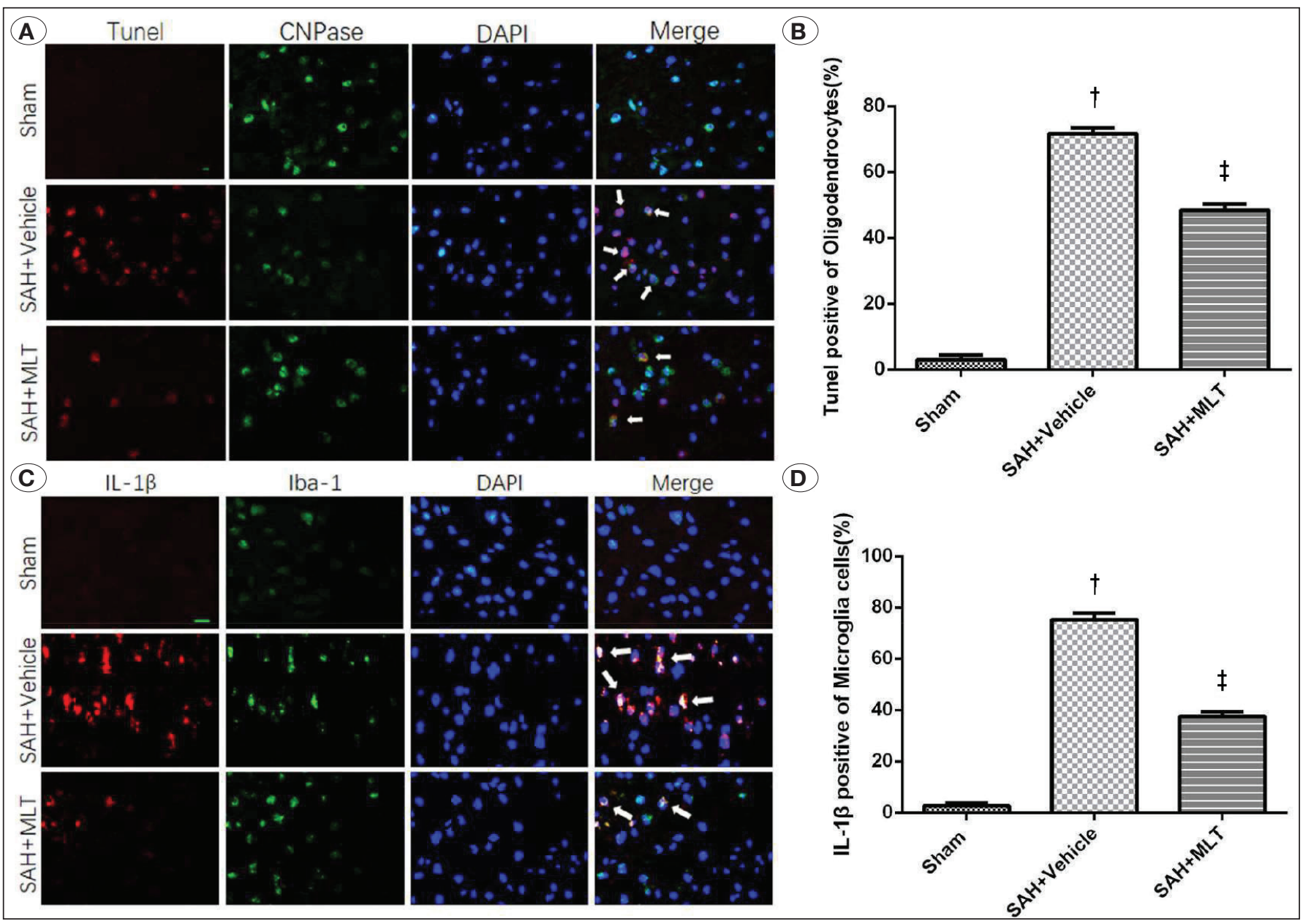

Figure 3: Protein levels of myelin basic protein (MBP), amyloid precursor protein (APP) accumulation, and expression of NOD-like receptor family pyrin domain-containing 3 (NLRP3) signal in the ipsilateral white matter. A-B) Representative Western blot images of MBP and APP demonstrating increasing APP and decreasing MBP in the subarachnoid hemorrhage (SAH) + vehicle group, and significant decreases after melatonin (MLT) treatment 24 hours (h) after SAH. C-D) NLRP3, caspase-1, and interleukin (IL)-1 $\beta$ expression levels were elevated at $24 \mathrm{~h}$. MLT treatment ameliorated these increasing trends. Density values of the blot were set to 1 (i.e., reference) in the sham group, and the other groups were compared with the sham group. 'Statistically significant difference (i.e., $p<0.05)$ compared with sham group; ¥Statistically significant difference (i.e., $\mathrm{p}<0.05$ ) compared with $\mathrm{SAH}+$ vehicle group.

vulnerable to injury by SAH. Diffusion tensor imaging (DTI) and histochemistry revealed typical pathological alterations including axonal injury in WM areas (18). Small-sample clinical experimental results suggested that DTI parameters, including fractional anisotropy and apparent diffusion coefficient, in WM areas are associated with SAH prognosis $(11,12)$. Our results revealed APP accumulation and loss of MBP in the WM after SAH, which may have been due to NLRP3 activation and downstream caspase- 1 and IL-1 $\beta$ in microglia and the induction of apoptosis in oligodendrocytes. A dose of 150 $\mathrm{mg} / \mathrm{kg}$ MLT inhibited NLRP3 expression, protected WM fibers, and improved neurological score, indicating that targeting WM protection may be potentially beneficial to patients who experience SAH.

MLT is a hormone produced by the pineal gland, which regulates sleep and wakefulness, and has been used for the treatment of insomnia in the clinic (26). Its amphipathic nature enables MLT to cross the BBB. Our recent study revealed that MLT could inhibit the NLRP3 inflammasome after stroke (5), and other experimental studies indicated that MLT ameliorates WM injury after neurological insult $(6,28)$. However, these data are still insufficient to explain how MLT regulates NLRP3 signal and protects WM. In the present study, we demonstrated that MLT inhibited the NLRP3 signal, as well as the downstream expression of caspase-1 and IL-1 $\beta$ in microglia in areas of WM. Microglia are essential inflammatory cells in the central nervous system after $\mathrm{SAH}$. A recent study reported that microglia accumulate in the WM after SAH; however, its acute impact remains elusive (29). Our data demonstrated NLRP3 signal activation in microglia and microglia accumulation in the WM post-SAH. MLT treatment alleviated oligodendrocyte apoptosis by altering Bim and Bcl-2. Future time-effect investigations may provide more evidence supporting the utility of MLT in clinical conditions. 


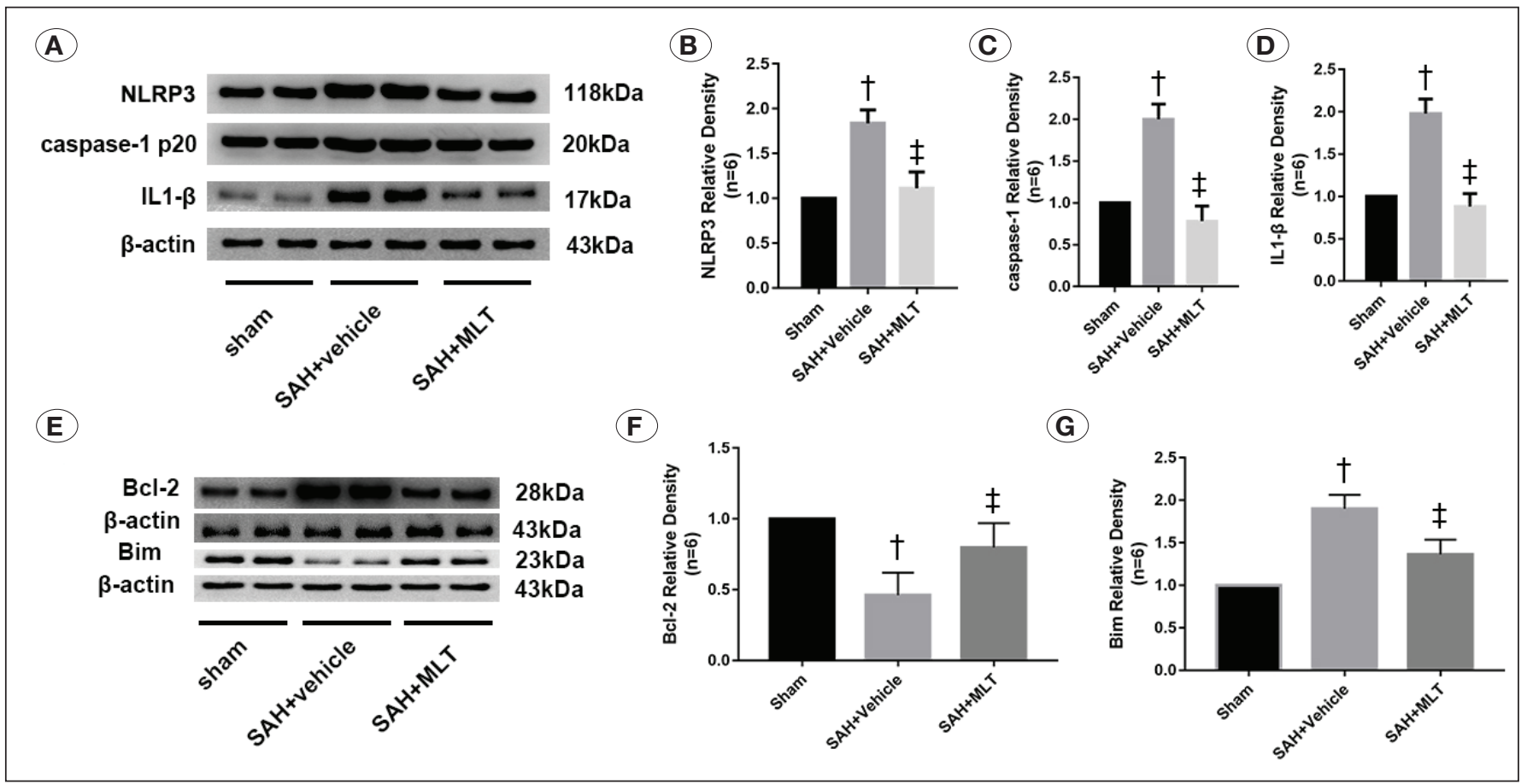

Figure 4: Protein levels of NOD-like receptor family pyrin domain-containing 3 (NLRP3) and apoptosis-related proteins in the ipsilateral white matter. A-D) Protein expression levels of NLRP3, caspase-1, and interleukin (IL)-1 $\beta$ are increased in mice in the subarachnoid hemorrhage (SAH) + vehicle group and reduced by melatonin (MLT) treatment $24 \mathrm{~h}$ after SAH. E-G) Protein expression levels of Bcl-2 and $\mathrm{Bcl}-2$-interacting mediator of cell death (Bim). Bcl-2 expression levels increased while Bim levels were reduced $24 \mathrm{~h}$ after induction of SAH. MLT treatment effectively reversed these trends. 'Versus sham group $(p<0.05)$; ${ }^{\ddagger}$ Versus $S A H+$ vehicle $(p<0.05)$.

Except for roles in anti-inflammation, MLT has also been reported to reduce neurological deficits in other conditions, such as acting as an anti-oxidant (1). MLT pretreatment profoundly reduced ischemia/reperfusion-induced autophagic flux in a mouse model of ischemic stroke. Furthermore, MLT ameliorated ischemia/reperfusion-induced neuronal apoptosis via the PERK/ATF4/CHOP pathway in endoplasmic reticulum stress (9). Recently, NLRP3 inflammasomes, which are critical for the formation of local inflammatory lesions, were found to be correlated with WM injury after induction of experimental multiple sclerosis (10). In a cuprizone model of demyelination, MLT dose-dependently decreased the mean number of apoptotic cells via caspase- 3 , Bax and $\mathrm{Bcl}-2$ apoptotic signaling (27). These phenomena may be inhibited NEK-7 NLRP3 interaction and downstream inflammation activation (15,31); nevertheless, further investigation should be conducted.

The present study had some limitations. Regarding the time course of drug administration, we investigated a one-time injection of MLT after SAH. A time-effect curve should be investigated in future basic studies. Regarding mechanism, we did not completely exclude other underlying molecular mechanisms associated with the protective effects of MLT on WM. Third, the upstream mechanism in which MLT influences NLRP3 signal, MBP, and APP after SAH remain incompletely elucidated. Therefore, more studies are needed to explain the exact mechanisms of WM protection by MLT before clinical use.

\section{CONCLUSION}

Results of the present study demonstrated that MLT attenuated EBI after SAH via NLRP3-induced apoptosis of oligodendrocytes. MLT exhibited the ability to cross the BBB in the central nervous system and has already been used in the clinic; therefore, it may be potentially beneficial to individuals who experience SAH.

\section{ACKNOWLEDGEMENTS}

This work was supported by the National Natural Science Foundation of China (Grant Nos. 81671174, 81671313, 81971133), LIAONING Science and Technology Project (2015020438, 20180550504). Medical Science Youth Breeding Project of Chinese People's Liberation Army (13QNP007), and China Postdoctoral Science Foundation (2015M572681).

\section{REFERENCES}

1. Alghamdi BS: The neuroprotective role of melatonin in neurological disorders. J Neurosci Res 96(7):1136-1149, 2018

2. Chen S, Feng $H$, Sherchan $P$, Klebe $D$, Zhao $G$, Sun $X$, Zhang J, Tang J, Zhang JH: Controversies and evolving new mechanisms in subarachnoid hemorrhage. Prog Neurobiol 115:64-91, 2014 
3. Chen Y, Luo C, Zhao M, Li Q, Hu R, Zhang JH, Liu Z, Feng $\mathrm{H}$ : Administration of a PTEN inhibitor BPV (pic) attenuates early brain injury via modulating AMPA receptor subunits after subarachnoid hemorrhage in rats. Neurosci Lett 588:131-136, 2015

4. Chen Y, Zhang Y, Tang J, Liu F, Hu Q, Luo C, Tang J, Feng H, Zhang JH: Norrin protected blood-brain barrier via frizzled-4/ beta-catenin pathway after subarachnoid hemorrhage in rats. Stroke 46:529-536, 2015

5. Dong Y, Fan C, Hu W, Jiang S, Ma Z, Yan X, Deng C, Di S, Xin Z, Wu G, Yang Y, Reiter RJ, Liang G: Melatonin attenuated early brain injury induced by subarachnoid hemorrhage via regulating NLRP3 inflammasome and apoptosis signaling. J Pineal Res 60:253-262, 2016

6. Drury PP, Davidson JO, Bennet L, Booth LC, Tan S, Fraser M, van den Heuij LG, Gunn AJ: Partial neural protection with prophylactic low-dose melatonin after asphyxia in preterm fetal sheep. J Cereb Blood Flow Metab 34:126-135, 2014

7. Egashira Y, Hua Y, Keep RF, Xi G: Acute white matter injury after experimental subarachnoid hemorrhage: Potential role of lipocalin 2. Stroke 45:2141-2143, 2014

8. Egashira Y, Zhao H, Hua Y, Keep RF, Xi G: White matter injury after subarachnoid hemorrhage: Role of blood-brain barrier disruption and matrix metalloproteinase-9. Stroke 46:29092915, 2015

9. Feng D, Wang B, Wang L, Abraham N, Tao K, Huang L, Shi W, Dong Y, Qu Y: Pre-ischemia melatonin treatment alleviated acute neuronal injury after ischemic stroke by inhibiting endoplasmic reticulum stress-dependent autophagy via PERK and IRE1 signalings. J Pineal Res 2017 (Ahead of print)

10. Fetisova E, Chernyak B, Korshunova G, Muntyan M, Skulachev $\mathrm{V}$ : Mitochondria-targeted antioxidants as a prospective therapeutic strategy for multiple sclerosis. Curr Med Chem 24:2086-2114, 2017

11. Fragata I, Alves M, Papoila AL, Ferreira P, Nunes AP, Moreira NC, Canhao P: Prediction of clinical outcome in subacute subarachnoid hemorrhage using diffusion tensor imaging. $J$ Neurosurg 2018 (Ahead of print)

12. Fragata I, Alves M, Papoila AL, Nunes AP, Ferreira P, CantoMoreira N, Canhao P: Early prediction of delayed ischemia and functional outcome in acute subarachnoid hemorrhage: Role of diffusion tensor imaging. Stroke 48:2091-2097, 2017

13. Fujii M, Yan J, Rolland WB, Soejima Y, Caner B, Zhang $\mathrm{JH}$ : Early brain injury, an evolving frontier in subarachnoid hemorrhage research. Transl Stroke Res 4:432-446, 2013

14. Gentleman SM, Nash MJ, Sweeting CJ, Graham DI, Roberts GW: Beta-amyloid precursor protein (beta APP) as a marker for axonal injury after head injury. Neurosci Lett 160:139-144, 1993

15. He Y, Zeng MY, Yang D, Motro B, Nunez G: NEK7 is an essential mediator of NLRP3 activation downstream of potassium efflux. Nature 530:354-357, 2016

16. Jang $\mathrm{SH}$, Chang $\mathrm{CH}$, Jung YJ, Kim SH, Seo JP: Optic radiation injury in patients with aneurismal subarachnoid hemorrhage: A preliminary diffusion tensor imaging report. Neural Regen Res 13:563-566, 2018

17. Kirkman MA, Allan SM, Parry-Jones AR: Experimental intracerebral hemorrhage: Avoiding pitfalls in translational research. J Cereb Blood Flow Metab 31:2135-2151, 2011
18. Kummer TT, Magnoni S, MacDonald CL, Dikranian K, Milner E, Sorrell J, Conte V, Benetatos JJ, Zipfel GJ, Brody DL: Experimental subarachnoid haemorrhage results in multifocal axonal injury. Brain 138:2608-2618, 2015

19. Li R, Ma K, Zhao H, Feng Z, Yang Y, Ge H, Zhang X, Tang J, Yin Y, Liu X, Tan L, Feng H: Cattle encephalon glycoside and ignotin reduced white matter injury and prevented posthemorrhagic hydrocephalus in a rat model of intracerebral hemorrhage. Sci Rep 6:35923, 2016

20. Macdonald RL, Schweizer TA: Spontaneous subarachnoid haemorrhage. Lancet (London, England) 389:655-666, 2017

21. Pan P, Zhang X, Li Q, Zhao H, Qu J, Zhang JH, Liu X, Feng H, Chen Y: Cyclosporine A alleviated matrix metalloproteinase 9 associated blood-brain barrier disruption after subarachnoid hemorrhage in mice. Neurosci Lett 649:7-13, 2017

22. Pang J, Peng J, Yang P, Kuai L, Chen L, Zhang JH, Jiang Y: White matter injury in early brain injury after subarachnoid hemorrhage. Cell Transplant 28:26-35, 2019

23. Sehba FA, Hou J, Pluta RM, Zhang JH: The importance of early brain injury after subarachnoid hemorrhage. Prog Neurobiol 97:14-37, 2012

24. Sugawara T, Ayer R, Jadhav V, Zhang JH: A new grading system evaluating bleeding scale in filament perforation subarachnoid hemorrhage rat model. J Neurosci Meth 167: 327-334, 2008

25. Swanson KV, Deng M, Ting JP: The NLRP3 inflammasome: Molecular activation and regulation to therapeutics. Nat Rev Immunol 19:477-489, 2019

26. Tan DX, Hardeland R, Manchester LC, Paredes SD, Korkmaz A, Sainz RM, Mayo JC, Fuentes-Broto L, Reiter RJ: The changing biological roles of melatonin during evolution: From an antioxidant to signals of darkness, sexual selection and fitness. Biol Rev Camb Philos Soc 85:607-623, 2010

27. Vakilzadeh G, Khodagholi F, Ghadiri T, Ghaemi A, Noorbakhsh F, Sharifzadeh M, Gorji A: The effect of melatonin on behavioral, molecular, and histopathological changes in cuprizone model of demyelination. Mol Neurobiol 53:4675-4684, 2016

28. Villapol S, Fau S, Renolleau S, Biran V, Charriaut-Marlangue $\mathrm{C}$, Baud O: Melatonin promotes myelination by decreasing white matter inflammation after neonatal stroke. Pediatr Res 69:51-55, 2011

29. Wu Y, Peng J, Pang J, Sun X, Jiang Y: Potential mechanisms of white matter injury in the acute phase of experimental subarachnoid haemorrhage. Brain 140:e36, 2017

30. Yuan J, Liu W, Zhu H, Zhang X, Feng Y, Chen Y, Feng H, Lin $\mathrm{J}$ : Curcumin attenuates blood-brain barrier disruption after subarachnoid hemorrhage in mice. J Surg Res 207:85-91, 2017

31. Zhang Y, Lv X, Hu Z, Ye X, Zheng X, Ding Y, Xie P, Liu Q: Protection of Mcc950 against high-glucose-induced human retinal endothelial cell dysfunction. Cell Death Dis 8:e2941, 2017

32. Zuo S, Li W, Li Q, Zhao H, Tang J, Chen Q, Liu X, Zhang JH, Chen Y, Feng $\mathrm{H}$ : Protective effects of Ephedra sinica extract on blood-brain barrier integrity and neurological function correlate with complement C3 reduction after subarachnoid hemorrhage in rats. Neurosci Lett 609:216-222, 2015 